\title{
Belén Salvatierra y la Psicología en Arequipa
}

\author{
Belen Salvatierra and the Psychology in Arequipa
}

\author{
Walter L. Arias Gallegos ${ }^{1}$ \\ Universidad Católica San Pablo
}

Recibido: $06-09-19$

Aceptado: $17-12-19$

\section{Resumen}

En este trabajo de corte histórico, se revisa y analiza la vida y obra de Belén Salvatierra, una de las psicólogas más destacadas del país. Su vida familiar, su formación profesional y su activa producción académica son nuestro objeto de estudio, dado que no existen trabajos previos que aborden las contribuciones que ha hecho a la psicología peruana. Se revisan sus libros, ponencias y artículos de investigación; así como su gestión como Decana de la Facultad de Psicología y Relaciones Industriales y Públicas que fortaleció la profesionalización de la carrera en Arequipa. También se revisan sus líneas de investigación en familia, neuropsicología y salud ocupacional.

Palabras clave: Belén Salvatierra; historia de la psicología; Arequipa.

\begin{abstract}
In this work, historical sort of, we review and analyze the life and work of Belen Salvatierra, one of the most relevant psychologist in our country. Her family, professional studies and her academic production are the target of this research, so there are not dado previous papers about her contributions given to the Peruvian psychology. We review her books, conferences and research articles; so as her management work as Dean of the Faculty of Psychology and Industrial Relations, which strength the process of professionalization of psychology in Arequipa. Moreover, we review her research in the lines of family studies, neuropsychology and occupational health.
\end{abstract}

Keywords: Belen Salvatierra; history of psychology; Arequipa.

\footnotetext{
1 Docente, Universidad Católica San Pablo. E-mail: warias@ucsp.edu.pe

(C) Los autores. Este artículo es publicado por la Revista de Investigación en Psicología de la Facultad de Psicología, Universidad Nacional Mayor de San Marcos. Este es un artículo de acceso abierto, distribuido bajo los términos de la licencia Creative Commons Atribucion - No Comercia_Compartir Igual 4.0 Internacional. (http://creativecommons.org/licenses/by-nc-sa/4.0/) que permite el uso no comercial, distribución y reproducción en cualquier medio, siempre que la obra original sea debidamente citada.
} 
Belén Salvatierra es una de las psicólogas más importantes del Perú, debido a su denodada labor profesional, académica e institucionalizadora, que sentó las bases para el desarrollo de la psicología regional en Arequipa. No solo ha sido merecedora de diversos premios y galardones que no hacen sino dar fe de su compromiso con la psicología y reconocer su trayectoria; pero además ha elevado el nivel de nuestra ciencia, cultivando áreas y temáticas que podemos decir, sin temor a equivocarnos, se encuentran mejor posicionadas académicamente en Arequipa, más que en ninguna otra parte del país. Quizá más importante aún, ha formado a diversas generaciones de psicólogos dentro de la senda del rigor científico y con una notoria preocupación por la trascendencia social de la profesión, a través de una profunda conexión con las necesidades de los pueblos y nuestra cultura.

En el presente trabajo analizamos su vida y obra destacando sus experiencias familiares y formativas, sus méritos académicos y profesionales, sus cargos y docencia universitarios, sus publicaciones y conferencias, sus aportes a la neuropsicología y la psicología ocupacional. Para ello, nos basamos tanto en fuentes primarias y en datos producto de varias entrevistas, en ambos casos, facilitados y coordinados con la venia de Belén Salvatierra, a quien le agradecemos enormemente por su tiempo, su confianza y su desinteresado apoyo.

Asimismo, al margen de la cercanía que podamos tener con la biografiada, hemos tratado de realizar en lo posible un trabajo objetivo en función de los datos obtenidos y de la lectura de su obra. Esperamos con ello, presentar un trabajo fiel a la verdad, que resalte los logros de Belén, pero también con un profundo sentido crítico que es propio de la actividad académica y de la historiografía de la psicología.

\section{Primeros años y vida familiar}

Belén Salvatierra nace el 2 de agosto de 1944 en la ciudad de Arequipa, hija de Zoila Rosa Salvatierra Miranda, quien emigró de Ecuador y que conoció al padre de Belén en Arequipa. En el colegio Rosario cursó sus estudios primarios y los estudios secundarios en el colegio de señoritas Arequipa donde se destacó en letras logrando la excelencia académica. Mientras estuvo en el colegio Arequipa, la profesora Amelia Díaz Valdivia cultivó en ella la pasión por las artes escénicas, y Belén se destacó rápidamente en el teatro, de modo que José Mejía Bejarano quien dirigía el Grupo Universitario de Teatro Experimental (GUTE) de la Universidad Nacional de San Agustín (UNSA) la invitó a formar parte del elenco, y puso en escena obras tales como El mancebo que casó con mujer brava y La fablilla del secreto bien guardado de Alejandro Casona y Larga despedida de Tennessee Williams. Sería en una de estas obras que conoció a Alberto Vega, reconocido poeta y pintor local, que además sería su futuro esposo (Ugarte y Chocano, 2014). 
En 1962 inició sus estudios de Educación en la Universidad Nacional de San Agustín, y fue no solo fue una destacada estudiante, sino también una mujer muy activa en el campo del arte y la política. Cuando estudiaba la carrera de Educación fue delegada del Centro Federado y dejó el teatro por el bordado. Este arte lo desarrolla gracias a su madre Zoila quien era experta en costura, hablaba francés y tocaba piano, arte que desarrolló de su padre -el abuelo de Belén- quien fabricaba pianos en Chile, su país natal. En 1965 presentó la II Exposición de Pintura Bordada en la galería del Banco Popular con el auspicio del Departamento Extensión Universitaria que crea Gustavo Quintanilla Paulet (19291999), personaje ilustre de Arequipa que desarrolló su obra académica en el campo de la filosofía y promovió el desarrollo de la cultura y la educación, fundando junto con el padre William Morris (1910-1999) la Universidad Católica de Santa María en 1961, donde llegó a ser profesor Emérito y regentó las primeras cátedras de psicología en dicha casa de estudios, como el curso de "Psicología general" que dictó en 1963 (Paredes, 2012).

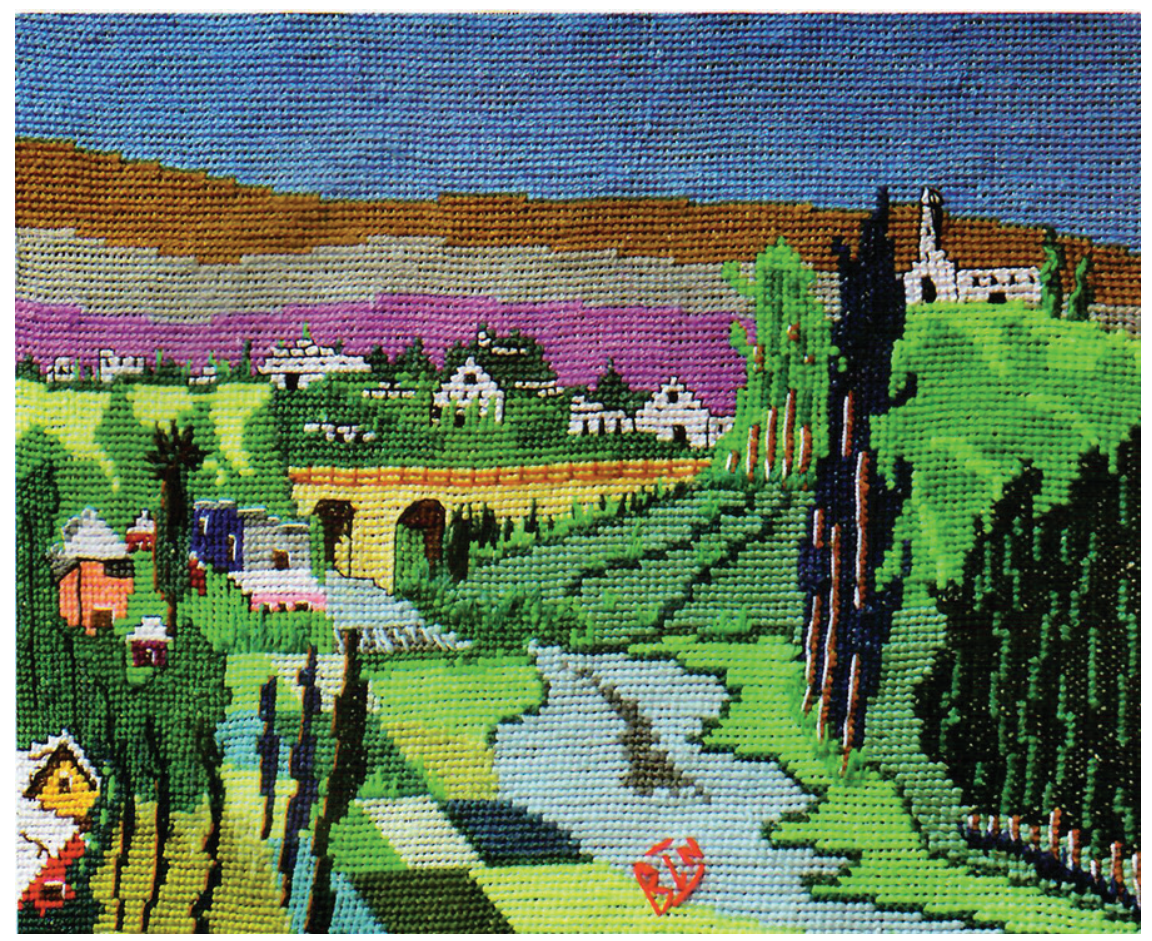

Figura 1. Bordado de Belén Salvatierra titulado "Puente Bolognesi" (38 x $51 \mathrm{~cm}$.) Fuente: Centro Cultural Chávez de la Rosa (2015, p. 18)

El arte bordado de Belén Salvatierra ha sido expuesto hasta en diez oportunidades, siendo la más reciente el año 2015, cuando por primera vez, se expusieron de manera conjunta los bordados de Belén y las acuarelas de Alberto 
Vega. Se trataba de paisajes de la campiña y la arquitectura tradicional de Arequipa que eran recreados en bordado y en pintura, tanto por Belén como por Alberto, respectivamente (Centro Cultural Chávez de la Rosa, 2015). El contacto entre ambos culminó en un feliz matrimonio que se celebró un martes 13 de diciembre de 1966, cuando Belén tenía 22 años. Siete años después tuvieron una hija, Zoila Elena Vega Salvatierra, que se ha destacado como musicóloga y como investigadora de la música arequipeña entre los siglos XV al XX. Ha recibido importantes reconocimientos, como el Premio a Tesis Doctoral otorgado por la Asamblea Nacional de Rectores el 2006 y el Premio de Novela Corta Julio Ramón Ribeyro organizado por el Banco Central de Reserva del Perú, también el 2006. Es profesora de la cátedra de Análisis e Investigación Musical en la Escuela de Artes de la UNSA y fue directora titular de la Orquesta Sinfónica de Arequipa del 2000 al 2012. Entre sus publicaciones figuran Texto y contexto de la obra de Roberto Carpio en la Arequipa del siglo XX el 2001, Vida musical cotidiana en la Arequipa del oncenio de Leguía 1919-1930 y Música en la catedral de Arequipa 1609-1881, el 2011 (Vega, 2011).

Belén se gradúa como educadora el 1966 con la tesis Afectividad y educación, en la que propone que si los estudiantes son estimulados a través de emociones positivas su rendimiento escolar será mejor. Con este trabajo se adelanta a las ideas más actuales que se han desarrollado en el seno de la Psicología Positiva, siendo uno de los pioneros Michael Fordyce que en la década del 1970 aplicó programas escolares para aumentar la felicidad de los estudiantes (Fordyce, 1977). Más recientemente dicha aproximación ha generado un cambio en la visión de la educación, la psicología y ciencias afines, con propuestas que se centran el desarrollo de la gestión de las emociones y la formación de virtudes, fortalezas y valores morales de los educandos, para maximizar sus recursos cognitivos y personales, consiguiendo con ello una formación más integral (Palomera, 2017).

Belén Salvatierra es parte de una generación de psicólogos que previamente fueron educadores o que bien cursaron en paralelo estudios de pregrado o postgrado en Educación, entre quienes podemos mencionar a Nicolás Paredes, Lucio Portugal, Dimas Quispe, Juan Zeballos, Helard Valdivia, etcétera. En ese sentido, Belén estudió psicología entre 1966 y 1970 en la Universidad Nacional de San Agustín de Arequipa, perteneciendo a las primeras generaciones de psicólogos graduados en Arequipa que promovieron de manera activa el desarrollo de la profesión y sentaron las bases para la labor investigativa futura.

\section{Actividad académica e institucionalización de la psicología arequipeña}

La UNSA fue la primera universidad de provincia donde se crea la carrera de psicología en el Perú, siendo la Universidad Nacional Mayor de San Marcos la primera donde se crea la carrera a nivel nacional en 1955 y la segunda en la Pontificia Universidad Católica del Perú en 1958, también en Lima (Alarcón, 2000). 
Aunque hemos podido rastrear los antecedentes más remotos de la psicología en Arequipa hasta 1848 con la obra frenológica de Juan Gualberto "Deán" Valdivia (Arias, 2018) y uno de los pioneros de la psicología peruana fue el arequipeño Honorio Delgado Espinoza (1892-1969), quien introdujo la farmacoterapia en el país y fue uno de los principales difusores del psicoanálisis en América Latina, que abogó por el desarrollo de la psicología y la formación psicológica de los profesionales de la salud (Arias, 2015), no fue hasta 1955 que inicia el proceso de institucionalización de la psicología arequipeña con la creación del Instituto de Filosofía y Psicología de la Facultad de Letras de la UNSA (Ballón, 1990).

Este instituto daría lugar a la Escuela Instituto de Psicología en 1959 y a la Escuela Profesional de Psicología en 1964 (Arias, 2016). Los cursos de psicología empero, figuraban desde 1931 con la cátedra de "Psicología experimental y filosofía lógica" que dictaba el Dr. Manuel Castañeda, y que fuera uno de los principales promotores de la creación del laboratorio de psicología experimental en la UNSA (Ballón, 1990), que después pasó a cargo de Félix Vargas Vinatea, filósofo que publicó uno de los primeros artículos psicológicos en la Revista de la Facultad de Letras de esta casa de estudios, sobre las sensaciones de los invidentes (Vargas, 1966). Otro pionero fue César Augusto Guardia Mayorga (1906-1983) quien en 1937 se incorpora a la UNSA como docente del curso "Historia de la filosofía antigua y metafísica", y en 1943 dicta los cursos de "Psicología general" y "Psicología del niño y el adolescente" (Jáuregui, 2005).

Al crearse la carrera de psicología en 1964, el director de la escuela sería el psiquiatra Carlos Neuenschwander Landa, que realizó sus estudios en Uruguay y fundó el Hospital Obrero en Arequipa en 1944 para dos años después crear el servicio de psiquiatría en dicho nosocomio. Se entrenó con Honorio Delgado y Carlos Alberto Seguín en Lima, y también recibió capacitación en Estados Unidos y París, por lo que se le puede considerar como el primer psiquiatra entrenado en Arequipa (Paredes, \& Arias, 2018). El Dr. Neuenschwander tuvo además, cierta presencia en la psiquiatría peruana, y aunque no ha sido si quiera mencionado en las obras sobre la historia de la psiquiatría peruana que publicó Oscar Valdivia (1964, 1989), participó en el III Congreso Latinoamericano de Psiquiatría en 1964 con la ponencia "Rasgos caracterológicos de la personalidad básica" (Neuenschwander, 1964) y en el Primer Congreso Nacional de Psiquiatría en 1969 con una ponencia sobre la enseñanza de la psiquiatría en Arequipa (Neuenschwander, 1969).

Otros psiquiatras que dictaron clases en la naciente Escuela Profesional de Psicología de la UNSA fueron Noel Altamirano, psiquiatra ayacuchano formado en la Universidad Nacional de Córdova que dictó el curso de "Psicología patológica" desde 1965; Marco Tulio Carpio Bernedo que estudió psiquiatría en la Universidad de La Plata y fue discípulo de Roberto Ciafardo (1908-1978), tuvo a su cargo las cátedras de "Diagnóstico psicológico" y "Terapéutica psicológica"; Claudio Mendívil se formó en Brasil y enseñó los cursos de "Bases biológicas de 
la mente" y "Psicología reflexológica" en 1965 y el curso de "Higiene mental" en 1967 (Paredes y Arias, 2018). Asimismo, la primera promoción de estudiantes de la carrera de psicología de la UNSA estuvo integrada por aproximadamente 50 estudiantes, muchos de los cuales habían seguido estudios previos en la Escuela Profesional de Educación de la UNSA (Paredes, 2014).

Para la segunda promoción, entre los psiquiatras que ejercieron la docencia se encuentran Hugo Delgado Flores (sobrino de Honorio Delgado), Paquita Valladares (que realizó la primera tesis de psicología en 1959 en la UNSA sobre el psicodiagnóstico de Rorschach), Elsa Rifal (formada en Brasil) y Ana María López Day (sobrina de Emilio Mira y López), entre otros profesores que fueron educadores como Julio Gómez Arias y filósofo como Félix Vargas Vinatea, Armando Barreda Delgado, etc. (Salas, 2014). Este fue el contexto en el que le tocó estudiar psicología a la Dra. Belén en la UNSA, un ambiente con una orientación marcadamente clínica, debido a la influencia de los docentes, quienes fueron psiquiatras y filósofos. Un ambiente, además, estimulado por el anhelo de la profesionalización de la carrera y el reconocimiento legal del quehacer del psicólogo, que significó, amén de los puntos de contacto con la psiquiatría y el aporte docente, una serie de disputas sobre la dependencia académica del psicólogo para con el psiquiatra, que fue bastante común en varios países de América Latina y generó diversos conflictos profesionales (Arias, 2011a), muchos de los cuales padeció la Dra. Belén, pues en una ocasión fue visitada de manera sorpresiva en su consultorio por un psiquiatra quien quería comprobar si en ese recinto se realizaban prácticas médicas, presumiblemente acusada por el celo que le tenían algunos psiquiatras locales dada la afluencia de pacientes que siempre tuvo desde que se inició en la práctica privada (Salvatierra, comunicación personal, 30 de junio de 2014).

Se graduó como psicóloga el 26 de setiembre de 1971 con la tesis titulada "El medio familiar y la adaptación del niño" y desde entonces se dedicó a la docencia universitaria. Como docente, la Dra. Belén preparó materiales de lectura y de trabajo para los estudiantes a quienes enseñó diversos cursos mientras ejerció las cátedras de Psicología educativa, Psicología de la motivación, Entrevista psicológica y Prácticas pre-profesionales de Psicología clínica y Psicología educativa en la Escuela Profesional de Psicología de la UNSA (véase Salvatierra, 1972, 1978, 1979a). Ese año, el 15 de abril de 1971, se fundó la Asociación de Psicólogos de Arequipa, de la cual Belén Salvatierra fue miembro junto con varios psicólogos que pertenecieron a las primeras promociones de psicología de la UNSA, y entre quienes figuran Nicolás Paredes Carpio, Dimas Quispe Yagua, Octavio Salinas Gutiérrez, Lucio Portugal Catacora, Dante Valdivia Zegarra, Juan Zeballos Carpio y Dora Zegarra de Vargas. La junta directiva estuvo conformada por Abraham Gonzáles Solís como presidente, Filiberto Bellido Vaca como vicepresidente, Helard Valdivia Ballón, como secretario general, Helder Zanabria 
Camacho como secretario de cultura, Modesto Chacón Mattos como secretario de economía, Helarf La Torre Calderón como secretario de prensa, Pedro Inga como secretario de investigaciones y Luis Vargas como secretario de asuntos gremiales.

En 1973, luego de haber estudiado en la Alianza Francesa, ganó una beca para seguir estudios de postgrado en el Instituto de Altos Estudios Escolares de la Sorbona, en París, en la especialidad de Psicología Escolar. Precisamente allí tomaría contacto con uno de los psicólogos más relevantes del siglo XX, René Zazzo (1910-1995), quien fuera discípulo de Henri Wallon (1879-1962), el principal promotor de la psicología escolar en Francia. Wallon organizó en 1919 la Primera Conferencia de Psicología Infantil y en 1948 fundó la revista Enfance, fue profesor del Instituto Nacional de Orientación Profesional que fundó Henri Piéron (1881-1964) en 1928, y presidió la Sociedad Francesa de Pedagogía en 1933 (Sos \& Esteban, 1998). Su pensamiento sobre el desarrollo del niño y la psicología infantil, estuvieron influidos por el marxismo, de hecho, Wallon fue profesor de uno de los psicólogos latinoamericanos que ha desarrollado la psicología marxista en campos como la psicología genética, la psicología pedagógica, la epistemología y la historia de la psicología: Alberto Leonidas Merani Colombo (Arias, 2011a).

Zazzo por otro lado, obtuvo su doctorado en la Sorbona y realizó estudios en Yale con Arnold Gesell (1880-1961). En 1950 sucede a Wallon en la conducción del Laboratorio de Psicología del Niño que formaba parte de la Escuela de Altos Estudios, donde Belén Salvatierra realizó sus estudios desde octubre de 1973 hasta mayo de 1974, ya que su hija Zoila contaba con pocos meses de nacida y tuvo que retornar a Arequipa. Sin embargo, su estancia por Francia le permitió conocer además de Zazzo, a Jean Piaget, la importante figura de la psicología evolutiva y el constructivismo pedagógico. Precisamente, La obra de Belén estuvo orientada por aquellos años hacia temas educativos, vocacionales y del desarrollo infantil, lo cual se evidencia en sus trabajos publicados en los años '70 y ' 80 .

En ese sentido, la Asociación de Psicólogos de Arequipa editó desde 1975 la Revista de Psicología en la que la aparecen varios artículos de investigación de la Dra. Belén, que corresponderían a sus primeras publicaciones académicas. Esta revista publicó hasta 1979 tres números, y sería la segunda revista de psicología del país (Arias, 2011b), ya que la primera se crea en 1959 y estuvo a cargo de la Sociedad Peruana de Psicología, entidad vinculada a la Asociación de Psicólogos de Arequipa (Arias y Caycho, 2019). Pues bien, en el primer número de 1975 se tienen siete artículos originales de los cuales el primero es de autoría de la Doctora Belén, y se avoca al análisis de la madurez para el aprendizaje en niños de edad preescolar, en el que evaluó a 96 niños de edad preescolar con el Test de Madurez para el Aprendizaje de J. Masey, reportando que las habilidades numéricas de los menores evaluados se encuentran disminuidas y que solo 3 niñas y 5 niños se encuentran en un nivel promedio de madurez (Salvatierra, 1975a). 


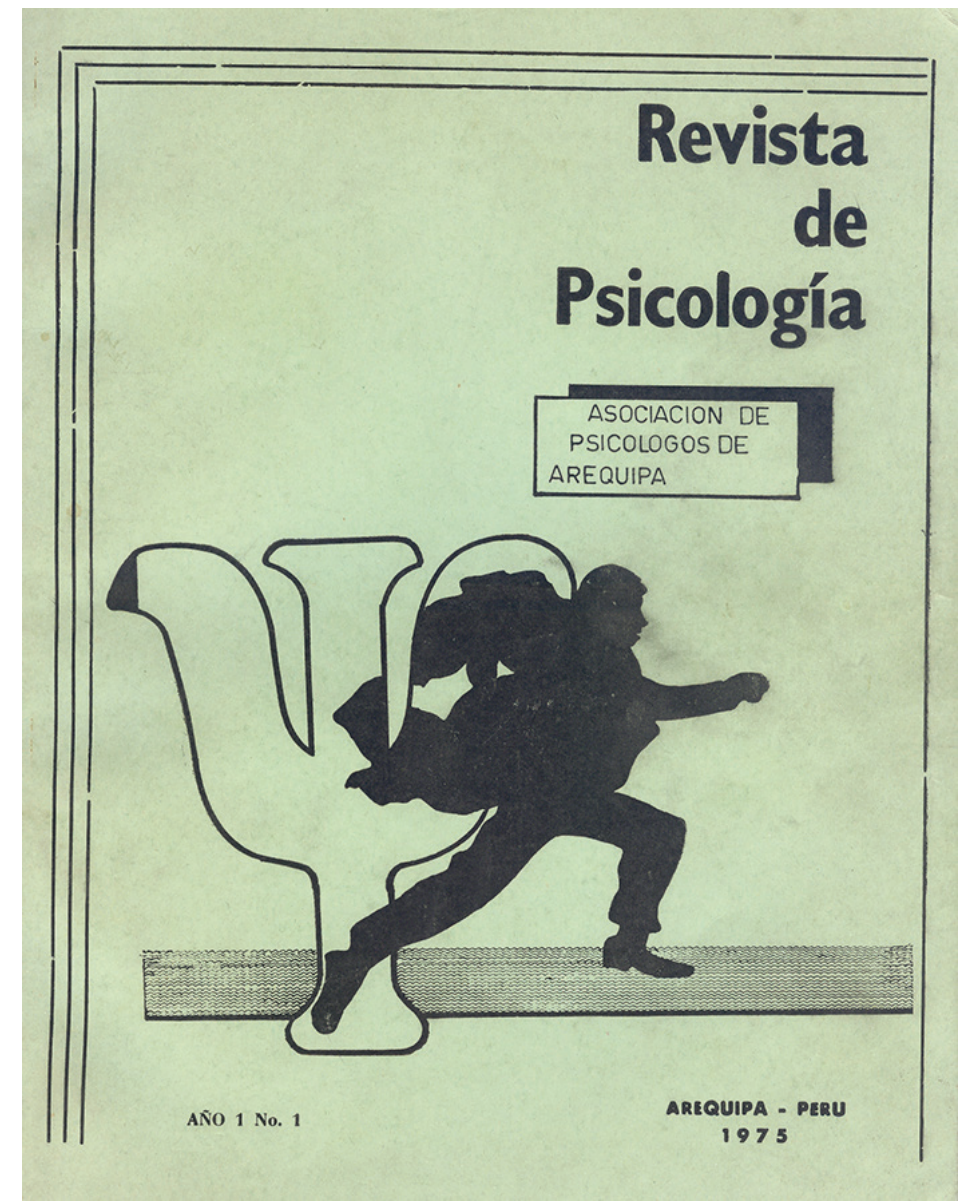

Figura 2. Revista de Psicología de la Asociación de Psicólogos de Arequipa Fuente: Archivo personal del autor

En 1976 se publicaron en un mismo volumen dos números de la Revista de Psicología de la Asociación de Psicólogos de Arequipa, en la que se tienen 10 artículos de investigación, siendo el último de autoría de la Dra. Belén, sobre un tema que ha sido objeto de otras publicaciones: la orientación vocacional. En este artículo, refuerza la necesidad de la orientación psicológica, más allá de la mera comunicación de los resultados de las pruebas vocacionales (Salvatierra, 1976). El tercer número se publicó en 1979 y aunque fue el último que se publicó, contó con artículos de Rubén Ardila y Emilio Ribes, representantes del conductismo en América Latina, así como de José Anicama y Tito Cuentas (1947-2019), reconocidos conductistas en Perú y Arequipa, respectivamente. También se publicó un artículo sobre los niños con síndrome de Down de autoría de Charles Portilla y Nicolás Paredes, otro artículo sobre los servicios de consejería en Estados Unidos escrito por Oscar Barreda y un estudio empírico acerca de la personalidad de los adolescentes 
varones que provienen de hogares monoparentales de autoría de Belén, en que se reporta que los menores evaluados presentan rasgos de agresividad, timidez y dependencia emocional de manera marcada (Salvatierra, 1979b).

Este artículo se alinea con un tema de estudio preferente por la Dra. Salvatierra, que ya se pudo apreciar en su tesis para obtener el título de psicóloga, y en el que por más de 20 años ha sentado un precedente hasta ahora inigualable. Nos referimos a la psicología de la familia, en el cual nuestra biografiada cuenta con una amplia producción. En ese sentido, el primer trabajo que publica la Dra. sobre familia data de 1977, el libro Guía práctica de psicología cotidiana; un texto escrito con lenguaje claro que brinda orientaciones sobre la formación de los niños en diversos aspectos tales como desarrollo socioemocional, educación y rendimiento escolar, psicosexualidad, relaciones parentales y conyugales, comunicación familiar, etc. Este texto, además, es muy ilustrativo sobre el trabajo del psicólogo en cuanto al campo de la orientación y consejería familiar, destacando la labor del psicólogo en la sociedad, contribuyendo a la desmitificación de su quehacer en el campo de la salud mental (Salvatierra, 1977).

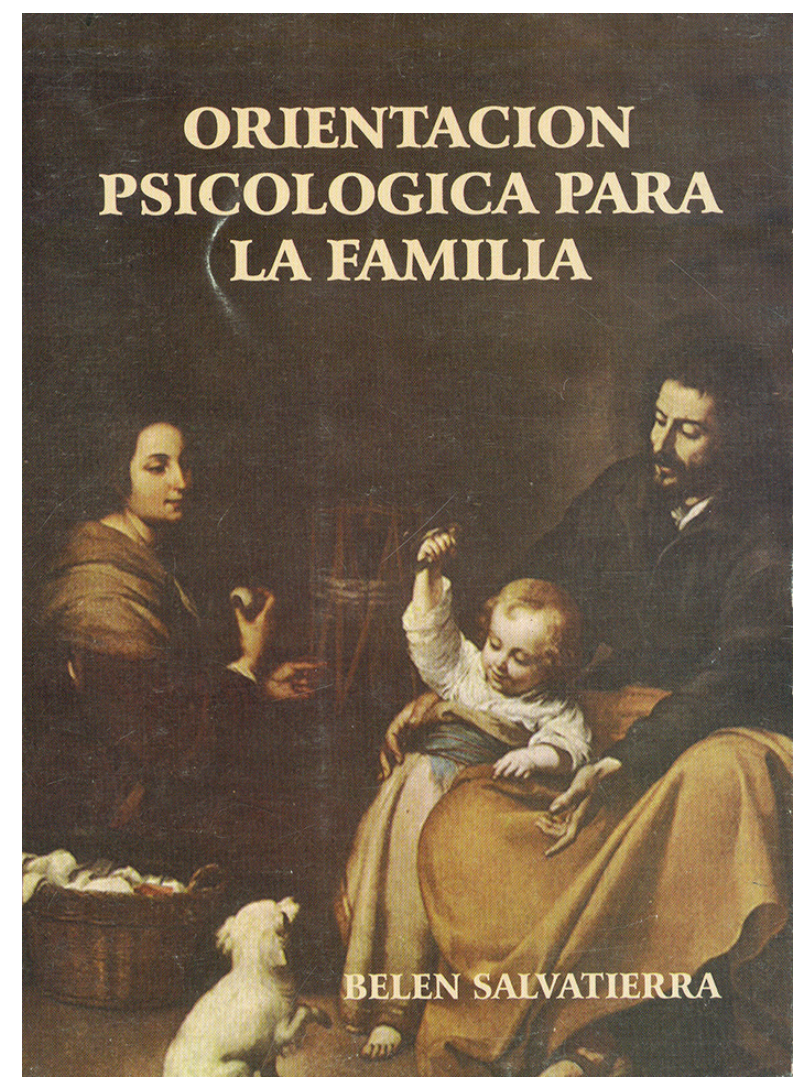

Figura 3. Portada del libro Orientación psicológica para la familia Fuente: Salvatierra (1997) 
Otros textos que han abordado temáticas similares son ¿Qué hago? (Salvatierra, 1992), El matrimonio (Salvatierra, 1993), Orientación psicológica para la familia (Salvatierra, 1997) y Cómo vivir la adolescencia sin padecerla (Salvatierra, 2004); libros todos ellos, que explican el correcto proceder en la educación y la crianza de los hijos en el hogar, analizando momentos críticos en el desarrollo del niño y brindando respuestas a interrogantes comunes que suelen hacer los padres de familia ante situaciones muy particulares como la masturbación infantil, el impacto psicológico del divorcio en los hijos, el consumo de drogas en los adolescentes, el castigo físico y sus repercusiones emocionales, etc. Los textos son llamativos por las ilustraciones que los amenizan, pero también por el abordaje que asume la autora en defensa de la familia y la objetividad con que trata cada uno de los temas, a la luz de los principios y teorías que ha desarrollado la psicología científica.

Otro medio a través del cual la Dra. Belén brindó orientación psicológica fue la televisión, pues entre 1975 y 1980, se emitió por canal Continental, un programa de televisión denominado "Consultando al psicólogo" (Salvatierra, 1986b). El programa salía al aire los martes y jueves desde las 18:30 horas hasta las 19 horas, en el que se abordaban los mismos temas que trataba en sus libros. Años después de la cancelación del programa, todos los contenidos estuvieron disponibles para la comunidad, pues fueron grabados en una colección de seis cassettes en formato VHS. El programa se canceló debido a que la Dra. Salvatierra viajó a México para estudiar su maestría en 1981; sin embargo, en 1980 realiza un viaje a Cuba donde realiza estudios en el Hospital Psiquiátrico de la Habana (Ugarte y Chocano, 2015) y participa del I Seminario Internacional de Psicología en la Comunidad, con una ponencia sobre las experiencias de orientación psicológica comunitaria por televisión (Salvatierra, 1980a), en alusión al programa que se transmitió durante cinco años en TV. Continental.

Durante tres años Belén Salvatierra estuvo en México, cursando una Maestría en Psicobiología en la Universidad Autónoma de México (UNAM), donde tuvo como profesores a importantes figuras de la psicología y las neurociencias mexicanas, como Víctor Colotla, Feggy Ostrosky y Augusto Fernández Guardiola, entre otros. Tenía 36 años y su hija Zoila siete años cuando se mudó a México y alquiló un departamento para vivir con su familia el tiempo que duró la maestría. Para aquel entonces, su esposo Alberto se había jubilado, y la práctica clínica a la que se dedicó de manera privada desde que obtuvo el título de psicóloga le permitió reunir el dinero suficiente para pagar sus estudios y radicar en México con su familia.

Su estancia en México le permitió formarse en neuropsicología y comprender las bases cerebrales de la conducta, además de aprender a utilizar los métodos neurofisiológicos de evaluación cerebral. Se graduó en 1983 con la tesis "Influencia de la amígdala del lóbulo temporal sobre los fenómenos fásicos del 
sueño en el gato (félix domésticus)" que se centra en el estudio del fenómeno del kindling amigdalino y la epilepsia experimental en gatos. Este estudio bien puede formar parte de la no corta tradición neuropsiquiátrica que se desarrolló en el Perú a mediados del siglo XX, dentro de la cual se destacan los estudios de Carlos Gutiérrez Noriega (1906-1950) sobre los efectos del cardiazol en los gatos (Gutiérrez, 1938), o la obra fundacional de Federico Sal y Rosas (1900-1974) de cuyas publicaciones el 90\% fueron sobre la epilepsia; además, en 1955 fue elegido por la Organización Mundial de la Salud como parte del Research Group for Juvenile Epilepsy y fundador de la Liga Peruana de la Lucha contra la Epilepsia (Valdivia, 1989).

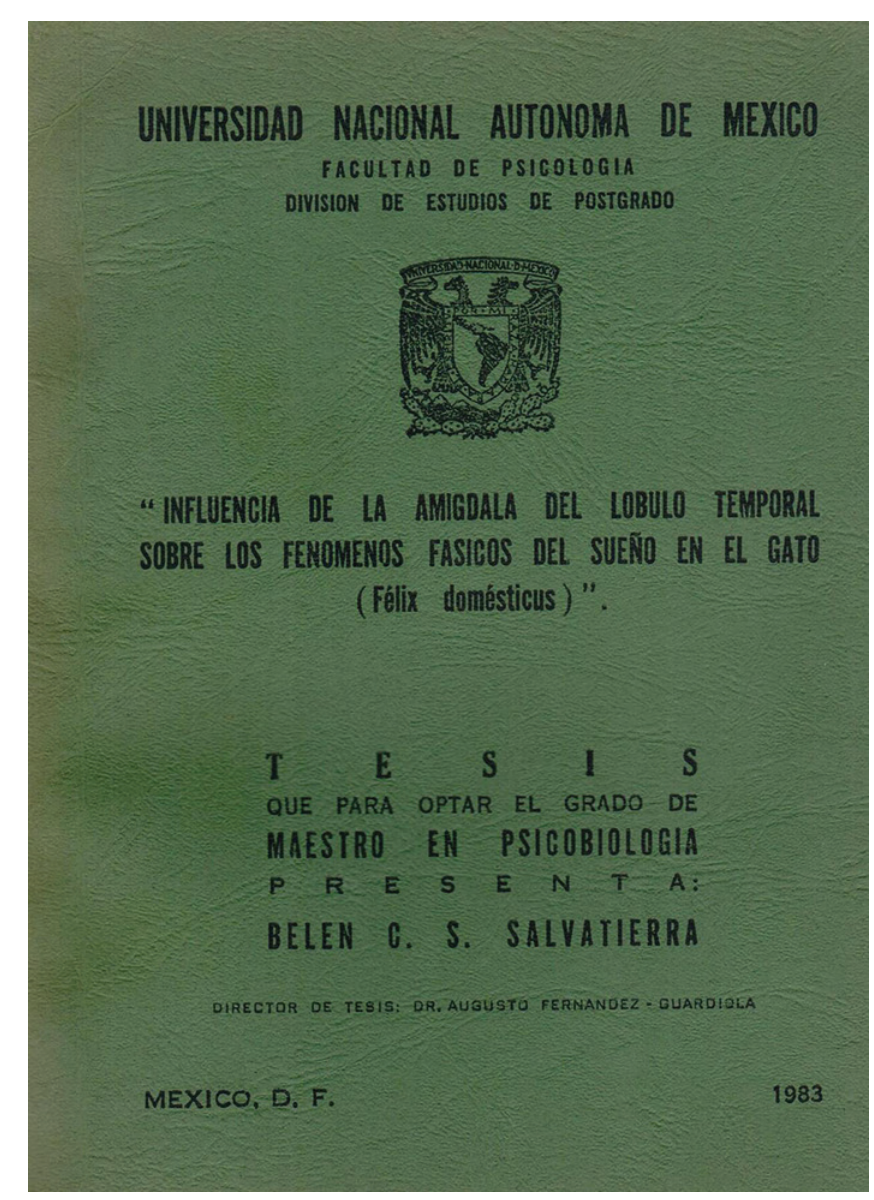

Figura 4. Portada de la tesis de maestría de Belén Salvatierra Fuente: Archivo personal del autor

Pues bien, este tema sería abordado por la Dra. Belén en su tesis doctoral, pero de ello hablaremos más adelante. Solo diremos que, a su regreso a Arequipa, 
se convirtió en la primera psicóloga con el grado de Magíster de la ciudad. Además, a finales de 1983 la UNSA formó una Comisión para realizar el estudio de factibilidad para crear la Facultad de Psicología, que estuvo integrada por Belén Salvatierra, Jorge Bellatín y Lucio Portugal; que el 27 de enero de 1984 presentó su informe sustentatorio que fue firmado por 22 psicólogos. Cabe señalar que para que pueda crearse la Facultad de Psicología se tuvo que unir a la Escuela Profesional de Relaciones Industriales y Públicas, que hasta entonces formaba parte de la Facultad de Derecho de la UNSA, de modo que se realizaron las gestiones para reunir estas dos escuelas en una sola Facultad, lo cual implicó fundamentar epistemológica y profesionalmente, dicha unión (Salvatierra, 2015). Finalmente, el 9 de marzo de 1984 se aprobó la creación de la Facultad de Psicología y Relaciones Industriales y Públicas (Arias, 2016). El 10 de abril se instaló el Consejo Universitario en el que se eligieron nuevos decanos, asumiendo el decanato de la flamante Facultad la Dra. Belén Salvatierra, mientras que en el Consejo de Facultad se eligió como Director de la Escuela Profesional de Psicología a Lucio Portugal Catacora (Portugal, 2014).

La Dra. Belén fue elegida decana en dos periodos consecutivos, el primero de 1984 hasta 1986 y el segundo desde 1987 hasta 1989. Durante este periodo, el laboratorio de psicología experimental estuvo a cargo de Filiberto Bellido como jefe y Renato Luza como asistente administrativo; asimismo, se crearon nuevas secciones, tales como la sala de educación especial, los ambientes de poligrafía y audiometría y el laboratorio de neuropsicología. En ese sentido, dada la formación en psicobiología de la Dra. Belén y el maltrato que recibían los estudiantes de psicología por parte de los profesores de la Escuela Profesional de Medicina que asumían las cátedras de Neuroanatomía, Neurofisiología y Neuropsicología; se cambiaron estas asignaturas por las de Neuropsicología I, II y III, respectivamente; que supusieron prescindir de la labor de los médicos (psiquiatras y neurólogos, principalmente) como docentes en la Escuela Profesional de Psicología de la UNSA. Ello también implicó que la Dra. Belén asumiera la coordinación de estas cátedras y se designara como profesores a psicólogos con conocimientos e interés en la neuropsicología como Dimas Quispe, Edgar Fernández y Julio César Salas (Salvatierra, 2015). Esta labor también implicó la elaboración de diversos materiales didácticos tales como guías, manuales, folletos y textos de trabajo; que eran distribuidos a los estudiantes con la finalidad de brindarles herramientas de estudio y orientación académica (Salvatierra, 1983b, 1986a, 1986b, 1989a, 1989b).

Durante este periodo la Escuela Profesional de Psicología de la UNSA, tuvo una importante presencia que se hizo notar en diversas actividades de carácter científico, tales como congresos, seminarios y simposios de psicología. En ese sentido, como ha sido destacado por Paredes (2016) la presencia de los psicólogos arequipeños en eventos de esta naturaleza ha sido siempre asidua, y la Dra. Belén ha sido ponente en varios congresos de psicología. Por ejemplo, en 1975 tiene lugar el Primer Congreso Peruano de Psicología organizado por la Sociedad 
Peruana de Psicología en la ciudad de Lima, y Belén Salvatierra participó con la conferencia "Un aporte a la orientación vocacional y profesional con la Batería Factorial de Aptitudes de J. M. Manzione, estandarizada en la ciudad de Arequipa" (Salvatierra, 1975b), pero en el libro de actas del congreso solo figura la ponencia de Octavio Salinas, a pesar de que participaron varios otros ponentes de Arequipa (Alarcón, Infante, Ponce, y Bibolini, 1976). También participó del Primer Congreso Regional de Psicología, con la ponencia "Riesgos de la enseñanza del segundo idioma en la primera infancia" (Salvatierra, 1980b). Este evento es muy particular, pues en el curso de este congreso, el 30 de abril de 1980 se promulgó la Ley $\mathrm{N}^{\circ} 23019$ que faculta la creación del Colegio de Psicólogos del Perú, y que fue recibida con júbilo por los asistentes. Otros eventos en los que participó la Dra. Belén, han sido el I Congreso Regional de Psicología con la ponencia "Un estudio con adolescentes usuarios ocasionales de cocaína" (Salvatierra, Quispe y Portugal, 1980), el I Seminario Regional de Psicología, con una ponencia sobre la ludoterapia (Salvatierra, 1981) y el I Congreso Peruano de Psicología Clínica organizado por el Colegio de Psicólogos del Perú en Arequipa, en el que la Dra. Salvatierra participó con dos ponencias: "La actividad póntico-genículo occipital (PGO) del sueño paradójico y su posible participación en la expresión e integración de las emociones" (Salvatierra, 1984) e "Importancia del apoyo de la familia del niño con retardo mental en los programas de aprestamiento de los programas de Educación Especial" (Riega y Salvatierra, 1984).

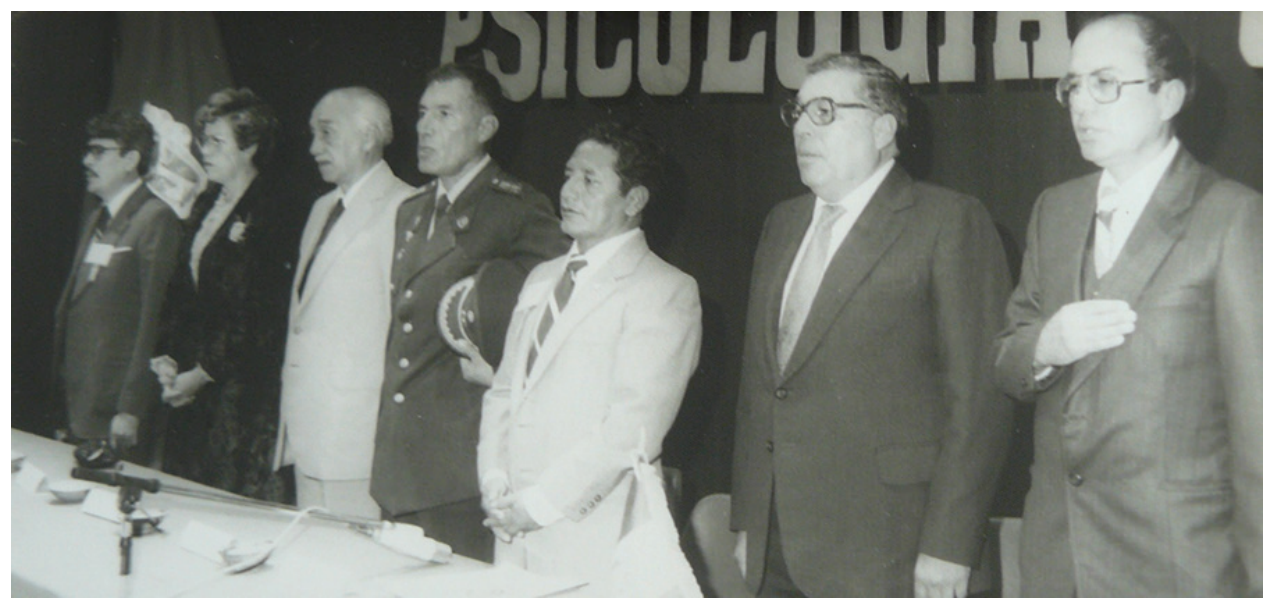

Figura 5. Inauguración del I Congreso Peruano de Psicología Clínica en 1984

Nota: De izquierda a derecha a parecen en esta foto Filiberto Bellido, Belén Salvatierra (Decana de la Facultad de Psicología y RR.II. de la UNSA), Dr. Manuel Zevallos Vera (Rector UNSA), representante de la Región Policial, Octavio Salinas, Decano del Colegio de Psicólogos del Perú y Abraham Gonzales.

Fuente: Archivo personal de Nicolás Paredes 
Asimismo, su visión académica también abarcó la labor editorial. En 1986, bajo la gestión de Belén como decana, se editó la primera revista de la Facultad que se llamaba Conciencia, y que solo llegó a editarse en dos ocasiones, contando con el apoyo del Concytec para su segunda edición (Arias, 2011b). En el primer número contó con 21 artículos, de los cuales dos fueron de escritos por profesores de la Escuela de Relaciones Industriales y Públicas, tres fueron ponencias de psicólogos en el I Congreso Nacional de Técnicas Psicoterapéuticas que tuvo lugar en Lima en 1982, 13 fueron artículos de revisión y solo tres fueron estudios empíricos: uno de autoría de Charles Portilla y Félix Jacobo sobre la sexualidad en adolescentes con retardo mental (Portilla y Jacobo, 1986), otro de Dimas Quispe sobre las dificultades del lenguaje en escolares (Quispe, 1986) y el tercero de autoría de Belén Salvatierra sobre el impacto del programa Consultando al psicólogo en una muestra de televidentes (Salvatierra, 1986b). El segundo número se editó en 1987con 11 artículos, de los cuales uno estaba firmado por un profesor de la Escuela de Relaciones Industriales y Públicas, otro era un artículo filosófico del otrora rector de la UNSA, alcalde provincial y presidente regional Juan Manuel Guillén Benavides; y el resto eran artículos escritos por los profesores de la Escuela de Psicología, de los cuales cuatro eran estudios empíricos: Uno trataba sobre la disfasia (Portilla, 1987), otro sobre el carácter de los políticos (Roldán, 1987), un tercer trabajo sobre el consumo de drogas de los reclusos del Penal de Socabaya (Hermoza, Pickman y Valvidia, 1987) y el cuarto trabajo analizaba el impacto de la enseñanza de una segunda lengua en la madurez para el aprendizaje escolar (Salvatierra, 1987). Una particularidad es que aproximadamente el 50\% de los contenidos respondían a una aproximación dialéctico materialista de psicología, corriente que ha sido predominante en la UNSA, y especialmente en la Escuela de Psicología, donde los estudiantes han sido formados siguiendo los modelos teóricos de la psicología soviética.

Otras de las actividades que se realizaron durante el decanato de Belén Salvatierra fueron la organización de la Segunda Especialidad en Psicología Educativa Orientación y Consejería Escolar en 1988 que culminó con 36 psicólogos titulados (Salvatierra, 1989c), quienes junto con la Dra. Belén como editora y autora principal publicaron el texto Guía para la Orientación Vocacional y Profesional (Salvatierra, 1991). Asimismo, la Facultad de Psicología y Relaciones Industriales y Públicas propuso la creación de un Centro Preuniversitario, que empezó a funcionar por primera vez desde 1987, y recibió la inscripción de 17,834 postulantes. También por primera vez se empleó un sistema computarizado de calificación que mereció el reconocimiento de la sociedad mistiana. En el área de proyección social, se fundó el Consultorio Psicológico que estuvo a cargo de Dante Valdivia, Angel Pickman, Ronald Hermoza y José Galdo Jarufe. También se organizaron anualmente, programas navideños para niños en situación de abandono o de sectores económicamente desfavorecidos de la ciudad. En estas actividades se montaba un show de títeres, les brindaba un desayuno y se les 
obsequiaba ropa y juguetes donados; llegando a congregar a hasta 1,500 niños en 1988 (Salvatierra, 2015).

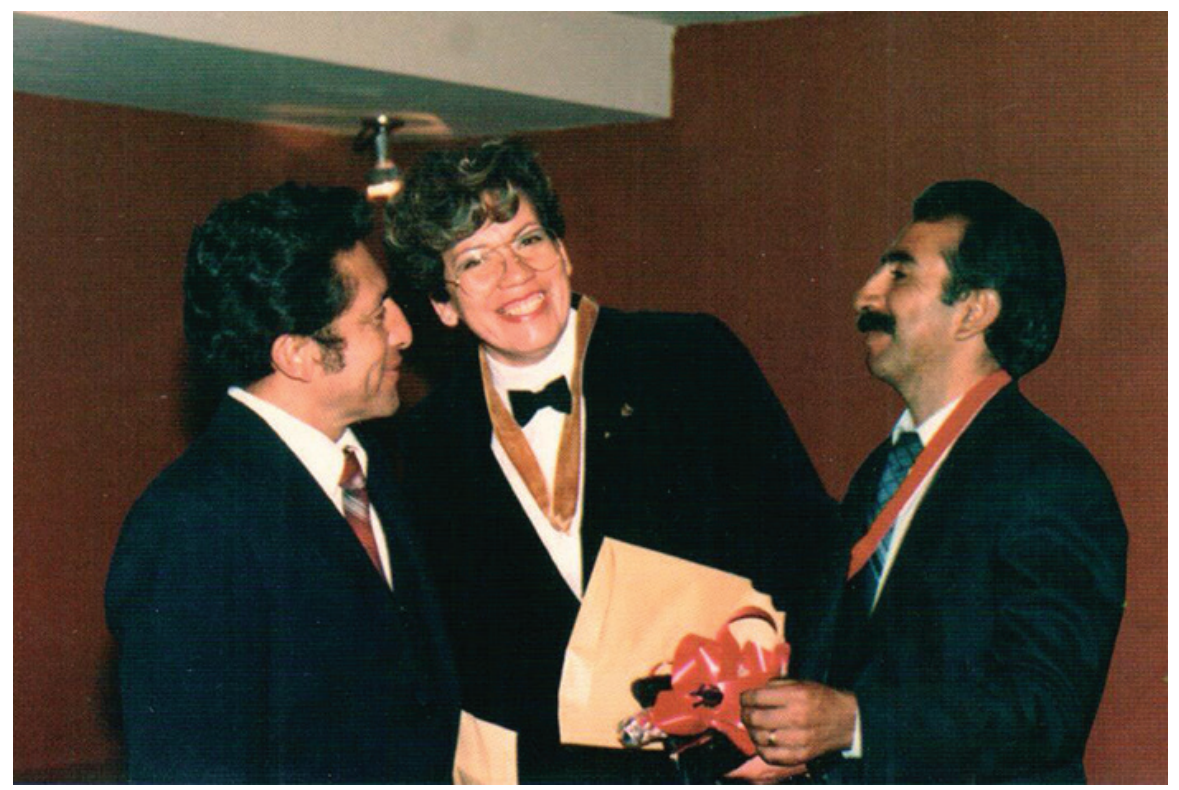

Figura 6. Dra. Belén Salvatierra recibiendo el nombramiento de Decana en su segundo periodo (1986)

Nota: La acompañan el entonces rector de la UNSA Dr. Juan Manuel Guillén Benavides y el Presidente del Jurado electoral Dr. Víctor Raúl Cadenas

Fuente: Ugarte y Chocano (2015, p. 21)

Durante su gestión como decana de la Facultad de Psicología y Relaciones Industriales y Públicas, Belén Salvatierra se caracterizó por su férrea convicción, su laboriosidad y por imponer una sólida disciplina académica, tanto a profesores como a estudiantes, que dio fruto en las actividades comentadas líneas arriba, y que favorecieron el proceso de institucionalización de la psicología regional. Por este motivo, fue apodada "la Tatcher", ya que también solía ser directa en sus intervenciones y confrontaba a las personas cuando algo era incorrecto. Jamás aceptó ninguna inconducta y fue severa para sancionar el mal proceder de algunos miembros de la comunidad universitaria. Esas características hicieron que ejerciera un liderazgo notable que llevó a la Facultad, y particularmente a la Escuela Profesional de Psicología de la UNSA a un reconocido posicionamiento académico en el país. Por ello, la década de 1980 ha sido denominada como la "época dorada" de la psicología en la UNSA que no ha vuelto a repetirse hasta la fecha, sino que, muy por el contrario, empezó un periodo de involución que dio pie al crecimiento y desarrollo de la psicología profesional en otras universidades de Arequipa (Arias, 2016). De este modo, en 1995 se creó la primera Escuela Profesional de Psicología en la Universidad Católica de Santa María, primera universidad privada y católica de Arequipa, donde bajo el liderazgo de Charles Portilla se orientó por cuatro ejes 
primordiales: la formación académica y profesional en pre y postgrado, la apertura de un servicio psicológico universitario, la creación del Consultorio de Atención Psicológica del Niño y el Adolescente (CAPNA), y la edición de la Revista de Psicología (Paredes, 2012). Por otro lado, la Universidad Católica San Pablo comenzó a ofertar la carrera de Psicología desde el 2007 con acento marcado en la investigación, de modo que a la fecha esta es la única escuela de psicología que cuenta con profesores investigadores acreditados por Concytec y fue la primera en obtener la acreditación en el sur del Perú (Palomino y Arias, 2018).

\section{Academia y profesión después de la UNSA}

Una vez que la Dra. Belén Savatierra culmina su relación académica con la UNSA, esto fue el 7 de agosto de 1989, cuando se jubila tras 20 años de servicio, inicia una nueva etapa de mayor especialización y producción, tanto en el terreno académico como profesional. Realiza un viaje a España en 1993 con la finalidad de averiguar sobre las oportunidades para hacer un doctorado en este país. De este modo, inicia sus estudios de doctorado en 1994 en la Universidad Complutense de Madrid, alcanzando el grado de Doctor en Psicología con el calificativo Suma Cum Laude. $\mathrm{Su}$ tesis fue dirigida por el Dr. Dionisio Pérez y Pérez, y se tituló "Aportaciones del electroencefalograma al diagnóstico y tratamiento neuropsicológico de las dificultades del aprendizaje" (Salvatierra, 1995). Durante su estancia doctoral tuvo como profesores a Juan Antonio Portellano y a Tomás Ortiz, ambas figuras importantes de las neurociencias de habla hispana, con una gran cantidad de publicaciones e investigaciones en su haber académico.

A su retorno a Arequipa, se le nombra Profesora Emérita de la UNSA en mérito a sus logros académicos y profesionales, así como por su entrega en la labor docente y su gestión como decana de la Facultad de Psicología y Relaciones Industriales y Públicas. Dos años después, Belén Salvatierra publica su tesis doctoral en formato de libro bajo el título "Niños con dificultades de aprendizaje" (Salvatierra, 1998) y que sería una guía para padres, maestros y psicólogos, que expone los resultados de su estudio y brinda diversas recomendaciones para evaluar y tratar a los niños con dificultades de aprendizaje, así como una serie de materiales para tales fines.

Ese mismo año, en 1998, el Colegio de Psicólogos del Perú, le otorga a Belén Salvatierra el Premio Nacional de Psicología y la invita como es menester dentro del marco de este premio, a ser conferencista principal del $I X$ Congreso Nacional de Psicología que tuvo lugar en Lima desde el 30 de setiembre al 2 de octubre de 1999. La Dra. Belén participó con una ponencia sobre un estudio que analiza el efecto que tiene la música en la afectividad de una muestra de músicos de la ciudad de Arequipa. Ese mismo año, en el cuarto volumen de la Revista Peruana de Psicología se publica como artículo dicha ponencia, con el título "Asimetrías cerebrales y la emotividad y susceptibilidad del músico". En ese 
sentido, la Dra. Belén ha sido una de las más dedicadas investigadoras peruanas en el campo de la neuropsicología, cuya obra no solo se remite a la ejecución y publicación de estudios en esta rama de la psicología, sino que también, como ya se dijo, fundó el laboratorio de neuropsicología de la UNSA y reformuló las cátedras de neuropsicología de esta casa de estudios, lo que dio lugar a la formación de una nueva generación de profesores con interés en neuropsicología, y que a su vez formaron a otras generaciones de psicólogos arequipeños que han conseguido abrirse camino en el campo de la academia internacional. Uno de estos investigadores sería Marcio Soto Añari, quien tiene varias publicaciones sobre reserva cognitiva y función ejecutiva en adultos mayores (Soto y Arcos, 2010; Soto y Belón, 2017; Soto y Cáceres, 2012; Soto, Flores y Fernández, 2013), además de haber formado un centro de investigación neuropsicológica, cuyos integrantes son ya catedráticos en diferentes universidades de Arequipa.

La Dra. Belén no ha dejado de hacer contribuciones en este campo, pues ha sido una importante colaboradora de la Revista de Psicología de Arequipa, publicación del Colegio de Psicólogos Consejo Regional III que se editó desde el año 2011 hasta el año 2017, con una periodicidad semestral y de manera ininterrumpida. Los artículos que han sido publicados por su autoría tratan diversos temas de su interés, tales como vocación, psicología del minero y neuropsicología. Así pues, en el tercer volumen de esta revista se editaron dos números dedicados a la neuropsicología, y la Dra. Belén participó con dos artículos, uno fue sobre su tesis de magíster (Salvatierra, 2013a) y otro sobre la detección neuropsicológica de las dificultades del aprendizaje en niños de edad preescolar (Salvatierra, 2013b). Otros trabajos suyos que se publicaron en la revista, fueron una revisión sobre la orientación vocacional y sus repercusiones en el mundo laboral (Salvatierra, 2011b) y un estudio sobre el perfil psicológico del minero peruano (Salvatierra, 2011a).

En ese sentido, uno de los temas que empezó a cautivar a la Dra. Salvatierra fue el de la psicología organizacional. Su interés por esta área de la psicología, comenzó a principios de los años 1990, cuando se retira de la UNSA y es invitada a dictar cursos de capacitación en Toquepala, Ilo, Cuajone, Ancash, Apurímac, Junín, Huánuco, Lima, etc.; para los trabajadores de las empresas Southern, Barrick, Buenaventura, Antamina, etc. Esta labor la llevó a publicar un texto de Orientación psicológica laboral (Salvatierra, 2000), que con el estilo de sus textos de orientación familiar, le permita concientizar a los trabajadores y directivos de las empresas mineras de la importancia que tiene la seguridad laboral y de su impacto en la productividad y el bienestar de los colaboradores, favoreciendo la prevención de accidentes laborales y la aparición de enfermedades ocupacionales. El texto comprende además del análisis de diversos factores que afectan la salud física y mental del trabajador, otros temas que resuenan actualmente dentro del campo laboral, pero que en nuestro país recién están siendo considerados dentro 
del marco legal de la salud y seguridad en el trabajo como lo estipula la Ley $\mathrm{N}^{\circ}$ 29783. Estos temas tienen que ver con la salud psicológica en el trabajo, el estrés laboral y los factores de riesgo psicosocial. Pero además toca temas tales como el civismo organizacional y los valores en la empresa, el conflicto familia trabajo y diversos aspectos psicológicos del trabajador como su autoestima, el carácter, las relaciones interpersonales en el trabajo, etc.

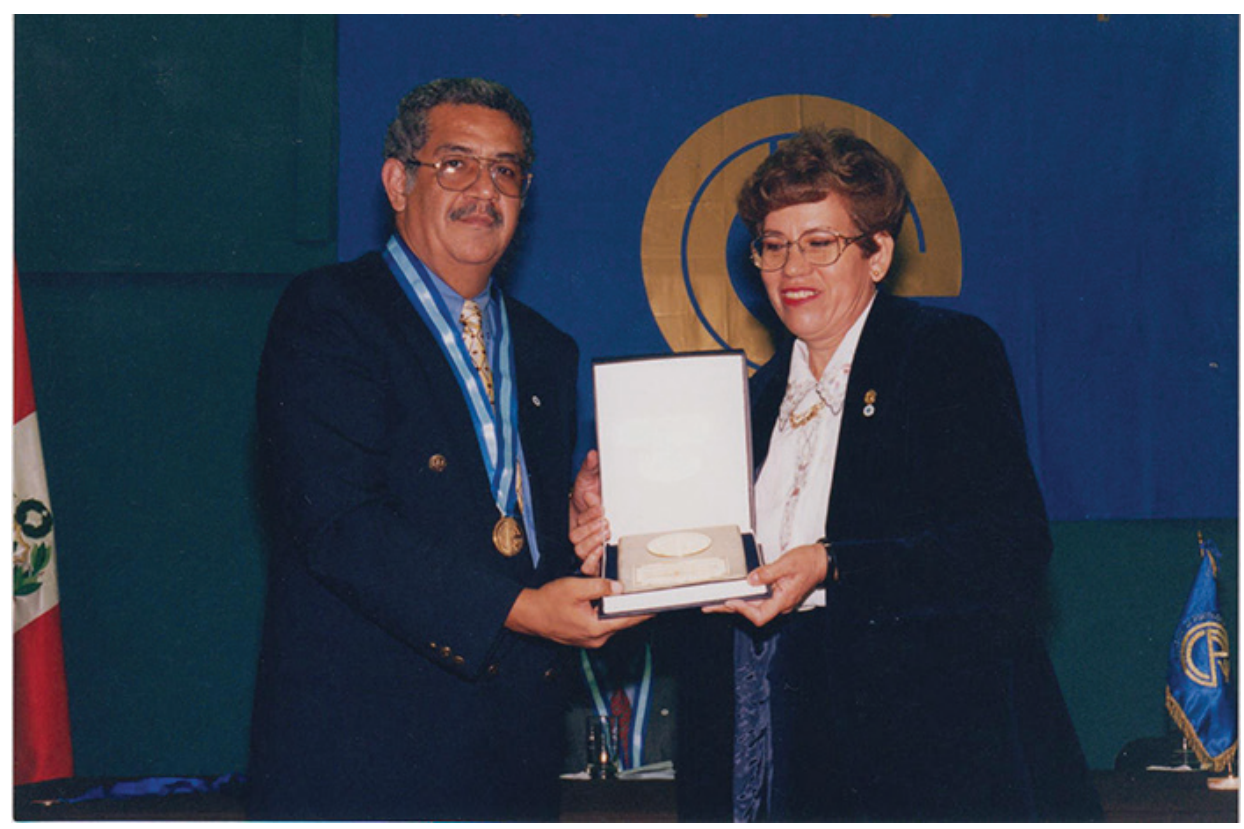

Figura 7. Dra. Belén Salvatierra recibiendo el Premio Nacional de Psicología (1998) de manos del Decano Nacional del Colegio de Psicólogos. Ps. Luis Zapata Fuente: Archivo personal de Belén Salvatierra

Por ello, la obra de Belén Salvatierra puede considerarse pionera en el campo de la salud mental ocupacional, pues existen pocos documentos relativos a estos tópicos en el Perú, si tenemos en cuenta que este trabajo de la Dra. Belén se desarrolló 20 años antes de la promulgación de la mencionada ley en el año 2012. Pero su incursión en la psicología organizacional no quedó en una propuesta orientadora y preventiva; sino que además de aplicar sus conocimientos psicológicos en las actividades propias de la psicología organizacional, como la selección de personal y la capacitación de los trabajadores, realizó importantes investigaciones durante una década, que constituyen una contribución relevante para la psicología organizacional y la salud ocupacional en el Perú.

$\mathrm{Y}$ es que, poco a poco, el abordaje de estos temas, le obligó a hacer evaluaciones a los trabajadores, en cuanto a algunas variables psicológicas que tenían cierta injerencia en su desempeño y su salud laboral. Un primer trabajo, 
sería el libro Psicología del minero (Salvatierra, 2001) en el que la autora estudia la problemática psicosocial del minero. En este texto se presentan una serie de orientaciones sobre la prevención de accidentes y el uso de equipos de seguridad, además de analizar una serie de hábitos saludables en el trabajador tales como el sueño, la alimentación y los aspectos psicoafectivos; dentro de los cuales se aborda la problemática familiar, pues como el minero labora lejos de su familia durante largos periodos de tiempo, eso lo hace proclive a desarrollar y mantener diversos vicios como el alcoholismo y la ludopatía, además de frecuentar centros de diversión nocturna, donde solicitan los servicios de damas de compañía, poniendo en riesgo su salud y perjudicando la armonía y la integración de su familia. Otro aporte digno de mencionarse es el estudio que realiza sobre el perfil psicológico de 651 mineros peruanos, concluyendo que su capacidad intelectual suele ser media o media baja y su grado de escolaridad mayoritariamente es de secundaria incompleta. Además, entre sus rasgos de personalidad se tiene la pasividad, la rigidez, la dependencia, busca la aprobación social y emocionalmente es poco estable.

Para el 2011, la Dra. Salvatierra publica un nuevo material: Cultura de seguridad laboral para el minero peruano (Salvatierra, 2011c). Este texto reúne varios estudios efectuados en mineros de todo el país, que abordan las presiones laborales y su impacto en las expresiones emocionales, la predisposición de los mineros a sufrir accidentes a partir de la valoración del clima organizacional y el pensamiento positivo, la motivación para usar implementos de seguridad, la implicancia de la asertividad en la prevención de riesgos laborales, la seguridad laboral a través del trabajo en equipos, y las relaciones humanas en la identificación de riesgos laborales. Todos estos temas son analizados siguiendo una metodología descriptiva y cuantitativa, con importantes sugerencias para los evaluados, pues en cada caso se especifican las características de la muestra, así como los instrumentos y procedimientos empleados. Asimismo, debe recalcarse que todas estas evaluaciones han sido acompañadas de charlas y talleres de sensibilización para los trabajadores, sus familias y los directivos; de ahí que no son solo datos crudos sobre la realidad del minero peruano, sino también una alternativa para su abordaje práctico sobre la base de la evidencia objetiva y concreta.

En cuanto a su participación en eventos académicos, podemos mencionar la ponencia "Detección neuropsicológica de dificultades de aprendizaje en niños de edad escolar" (Salvatierra, 2003) presentada en el I Congreso Nacional de Psicología Clínica - Educativa que organizó el Colegio de Psicólogos Consejo Directivo Regional III de Arequipa. Asimismo, en el I Congreso Internacional de Psicología organizado por la Universidad Católica de Santa María, presentó la conferencia magistral "La dislexia a la luz de la neuropsicología evaluativa y la biocibernética" (Salvatierra, 2006), y en el XVI Congreso Peruano de Psicología, organizado por el Colegio de Psicólogos del Perú, expuso sus experiencias de su 
trabajo con los mineros peruanos, bajo el título "El quehacer del psicólogo en la minería del siglo XXI" (Salvatierra, 2013c).

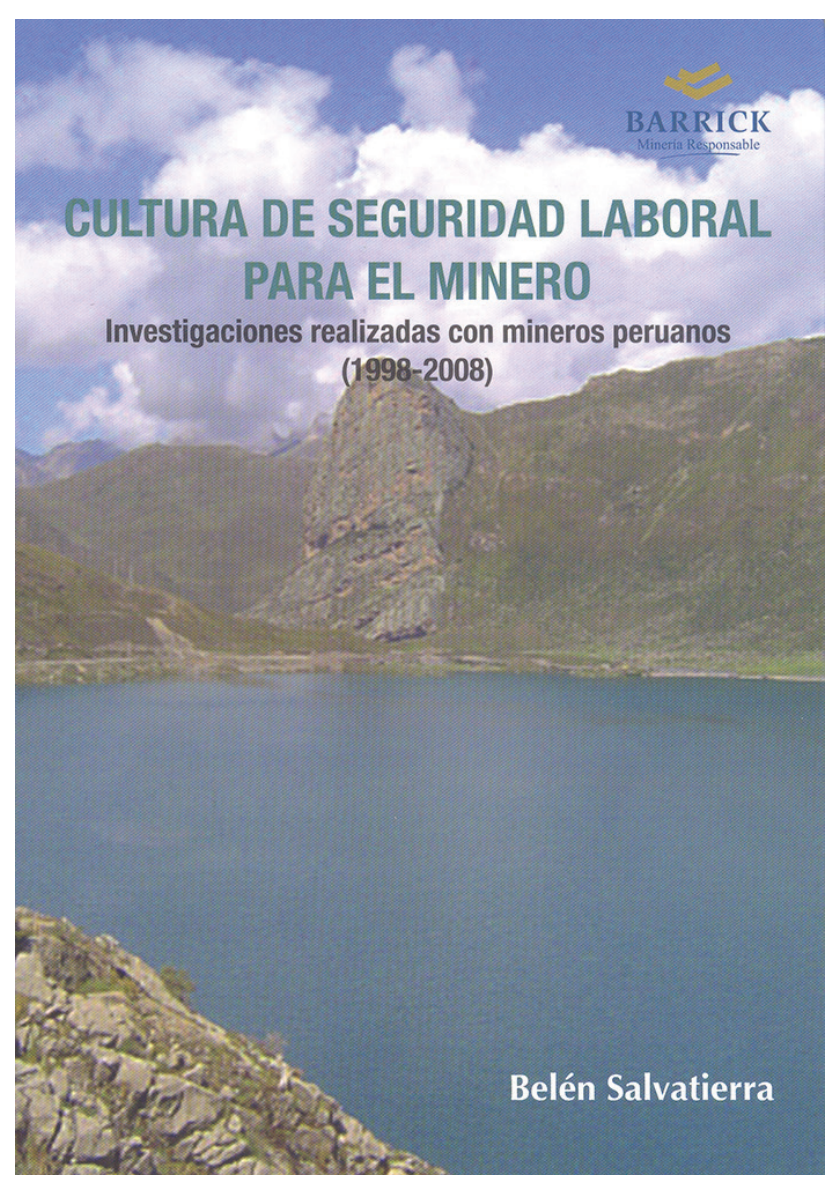

Figura 8. Portada del libro Cultura de seguridad laboral para el minero Fuente: Salvatierra (2011c)

Entre otras actividades, la Dra. Belén, ha viajado invitada a diversas ciudades de países de Latinoamérica como Chile y Argentina, para explicar la relevancia de la psicología en el campo laboral de la minería, a la luz de sus estudios y el despliegue profesional que durante 20 años tuvo en el campo de la psicología organizacional. Asimismo, aunque no ha dictado más cátedra en pregrado, sí ha sido profesora en maestrías y doctorados. También se ha dedicado a la consulta privada, tanto en el país como en el extranjero (México, Argentina, Chile, Francia y España), actividad que no ha dejado desde que obtuvo el título de psicóloga en 1971, y que además, le permitió ganar una bien merecida fama de profesional competente, experta psicoterapeuta y sobre todo, un ser humano íntegro en todo el sentido de la palabra. En consecuencia, el Colegio de Psicólogos Consejo 
Directivo Regional III de Arequipa le otorgó un significativo reconocimiento por su trayectoria profesional el año 2001, conmemorando su trayectoria profesional y sus esfuerzos por desarrollar la psicología.

En ese sentido, algunos rasgos de su quehacer profesional son la autoridad de su experiencia y formación como psicóloga y educadora; que decantan en su praxis, a través de un discurso integrador, que combina coherentemente sus conocimientos, sus valores y su pasión por una alta calidad en el desempeño profesional. Ella nunca dirá algo que no siente, piensa o cree; siempre será directa y estará del lado de la verdad y la justicia. Científicamente, ha sido una infatigable investigadora, cuyo trabajo ha cimentado el desarrollo de la Psicología en Arequipa y ha promovido líneas de investigación que hoy gozan de solidez académica en la región, como son la psicología educativa, la psicología de la familia, la neuropsicología y la psicología organizacional. Campos todos estos, en los que ha sabido imprimir su visión dialéctica del hombre y del mundo, con la gran ventaja que le da el haber trascendido al discurso propio de la psicología marxista, dándole sustento en el quehacer investigativo y la transparencia de sus acciones, siempre orientadas por el bien de los demás, al margen de sus intereses personales.

\section{En perspectiva}

Belén Salvatierra es sin lugar a dudas, una de las psicólogas más importantes de Arequipa, como lo acredita un estudio previo en el que los estudiantes de cuatro universidades de la ciudad, la designaron como una de las psicólogas con mayor relevancia en el campo profesional y académico a nivel local y nacional (Arias, Arista, Choque, Angles, Chávez y Herrera, 2015). Su labor abarca no solo la docencia y la práctica clínica, que ha sabido ejercer con solvencia académica y moral; su obra ha favorecido la institucionalización de la psicología arequipeña, dándole a través de su gestión como decana y sus trabajos investigación, rasgos muy particulares, que aún hoy se mantienen, como las líneas de trabajo en familia, neuropsicología y salud laboral; por parte de nuevas generaciones de psicólogos.

Ha sido también una constante colaboradora en diversos proyectos académicos como la Asociación de Psicólogos de Arequipa y las revistas de psicología que se han editado en la región. Su participación en los diversos certámenes científicos a los que ha acudido como expositora, han dejado una grata impresión del desarrollo que ha alcanzado la psicología en provincias. Su prolífica producción a través de los libros que ha publicado durante poco más de 40 años, no ha sido superada por ningún psicólogo arequipeño. Todo ello le ha valido los más importantes reconocimientos que se puede dar a un psicólogo peruano: el nombramiento como Profesora Emérita de la UNSA, el Premio Nacional de Psicología y el reconocimiento a su trayectoria, son solo algunos de los galardones que ha recibido. 
Más importante aún, es la solidez de sus ideas y la claridad de su pensamiento, que han servido de estímulo para las no pocas generaciones de psicólogos que se han formado en la UNSA. Su tránsito por diversas áreas de la psicología, han mostrado la relevancia de la profesión y el impacto favorable que puede tener el psicólogo en la salud, la educación y el trabajo. Estamos pues, ante una profesional que ha sido pionera en diversos sentidos: primera psicóloga magíster en Arequipa, primera decana de la Facultad de Psicología y Relaciones Industriales y Públicas, primera psicóloga arequipeña que ha recibido el Premio Nacional de Psicología, y primera psicóloga arequipeña que ha realizado tanto investigación neuropsicológica como en el campo de la salud laboral.

\section{REFERENCIAS}

Alarcón, R. (2000). Historia de la psicología en el Perú. De la Colonia a la Republica. Lima: Universidad Ricardo Palma.

Alarcón, R., Infante, J., Ponce, P., y Bibolini, A. (Eds.) (1976). La Investigación psicológica en el Perú. Lima: Sociedad Peruana de Psicología.

Arias, W. L. (2011a). Líneas comunes en la historia de la psicología latinoamericana. Revista de Psicología de Arequipa, 1(1), 27-47.

Arias, W. L. (2011b). Revistas de psicología en Arequipa. Nuevos Paradigmas, 5(2), 59-84.

Arias, W. L. (2015). Honorio Delgado (1892-1969), un repaso histórico sobre su vida y su obra: A propósito de los 100 años del psicoanálisis en el Perú. Boletín de la Academia Paulista de Psicología, 35(89), 286-308.

Arias, W. L. (2016). Contribuciones para una historia local de la psicología en Arequipa. En Mardones, R. Historia local de la psicología. Discusiones teóricas, metodológicas y experiencias de investigación (pp. 354-388). Chile: Universidad Santo Tomás.

Arias, W. L. (2018). Los estudios frenológicos de Juan Gualberto "Deán" Valdivia en la Arequipa del siglo XIX. Arequipa: Adrus - SPHP.

Arias, W. L., Arista, M., Choque, M., Angles, U., Chávez, P. y Herrera, B. (2015). Una encuesta acerca de los psicólogos más representativos y el futuro de la psicología en Arequipa. Liberabit, 21(1), 123-139.

Arias, W. L., y Caycho, T. (2019). La Revista de Psicología de la Sociedad Peruana de Psicología. Manuscrito sometido a revisión.

Ballón, H. (1991). Ensayos de sociología. Arequipa: UNSA.

Centro Cultural Chávez de la Rosa (2015). Pintura bordada y acuarela. Arequipa: Biblioteca Regional Mario Vargas Llosa.

Fordyce, M. W. (1977). Development of a program to increase personal happiness. Journal of Counseling Psychology, 24(6), 511-521.

Gutiérrez, C. (1938). Acerca del origen y mecanismo de las convulsiones producidas por el cardiazol en los gatos descerebrados. Revista de Neuropsiquiatría, 1(3), 373-419. 
Hermoza, R., Pickman, A. y Valdivia, H. (1987). Problemas de los farmacodependientes presos en las cárceles de Arequipa. Conciencia, 2(2), 43-49.

Jáuregui, F. (2005). Concepción filosófica de César Augusto Guardia Mayorga. (Tesis de Maestría). Universidad Nacional Mayor de San Marcos, Lima, Perú.

Neuenschwander, C. (1964). Rasgos caracterológicos de la personalidad básica. En C. A. Seguín y R. Ríos, Anales del III Congreso Latinoamericano de Psiquiatría (pp. 337340). Lima: Asociación Psiquiátrica de América Latina.

Neuenschwander, C. (1969). Contenido de la enseñanza y curriculum académico. En O Valdivia y A. Péndola, Psiquiatría Peruana (pp. 181-184). Lima: Asociación Psiquiátrica Peruana.

Palomera, R. (2017). Psicología positiva en la escuela: un cambio con raíces profundas. Papeles del Psicólogo, 38(1), 66-71. doi: 10.23923/pap.psicol2017.2823

Palomino, A. A. y Arias, W. L. (2018). La Revista de Psicología de la Universidad Católica San Pablo (2011-2018): Un análisis histórico y bibliométrico. Revista Peruana de Historia de la Psicología, 4, 41-64.

Paredes, N. (2012). Notas para una historia de la psicología en la Universidad Católica de Santa María. Revista de Psicología de Arequipa, 2(2), 131-141.

Paredes, N. (2014). Los inicios de la formación de psicólogos en la Universidad Nacional de San Agustín. Psicología de Hoy, 1, 12-16.

Paredes, N. (2016). Congresos y otros eventos científicos de psicología realizados en Arequipa (1975-2008). Revista Peruana de Historia de la Psicología, 2, 53-71.

Paredes, N. y Arias, W. L. (2018). El aporte docente de los psiquiatras a la psicología: cuatro personajes a la luz de la historia de la psicología en Arequipa. Revista de Neuropsiquiatría, 81(1), 29-41.

Portilla, Ch. (1987). Disfasia. Conciencia, 2(2), 23-35.

Portilla, Ch. y Jacobo, F. (1986). Sexualidad en adolescentes con retardo mental. Conciencia, 1(1), 12-22.

Portugal, L. (2014). Escuela de Psicología de la Universidad Nacional de San Agustín. Memorias y reflexiones 1964-1984. Arequipa: Editorial UNSA.

Quispe, D. (1986). Dificultades del lenguaje en niños escolares de la ciudad de Arequipa. Conciencia, 1(1), 68-79.

Riega, E., y Salvatierra, B. (1984). Importancia del apoyo de la familia del niño con retardo mental en los programas de aprestamiento de los programas de Educación Especial. Ponencia presentada en el I Congreso Peruano de Psicología Clínica, 3 - 7 de diciembre, organizado por el Colegio de Psicólogos del Perú, Arequipa, Perú.

Roldán, J. A. (1987). Necesidad de la orientación psicológica en el líder político. Conciencia, 2(2), 76-88. 
Salas, J. C. (2014). La recursividad de la escuela Profesional de Psicología en su quincuagésimo aniversario (1964-2014). Psicología de Hoy, 1, 9-11.

Salvatierra, B. (1972). Apuntes de psicología de la motivación. Arequipa: UNSA.

Salvatierra, B. (1975a). Un estudio sobre la madurez para el aprendizaje en niños de edad pre-escolar. Revista de Psicología de la Asociación de Psicólogos de Arequipa, 1(1), $1-12$.

Salvatierra, B. (1975b). Un aporte a la orientación vocacional y profesional con la Batería Factorial de Aptitudes de J. M. Manzione, estandarizada en la ciudad de Arequipa. Ponencia presentada en el I Congreso Peruano de Psicología, 1-5 de diciembre, organizado por la Sociedad Peruana de Psicología, Lima, Perú.

Salvatierra, B. (1976). ¿Para estudiar una carrera superior basta el rendimiento óptimo de una prueba de aptitudes aplicada en último momento? Revista de Psicología de la Asociación de Psicólogos de Arequipa, 2(1), 82-84.

Salvatierra, B. (1977). Guía práctica de psicología cotidiana. Arequipa: Editorial Tejada.

Salvatierra, B. (1978). Problemática psicosocial del adolescente. Arequipa: Arequipa.

Salvatierra, B. (1979a). Psicología educativa. Arequipa: UNSA.

Salvatierra, B. (1979b). Un estudio con adolescentes varones procedentes de hogares sin padre. Rasgos de personalidad más comunes. Revista de Psicología de la Asociación de Psicólogos de Arequipa, 3(1-2), 47-72.

Salvatierra, B. (1980a). Experiencia de orientación psicológica comunitaria por televisión. Ponencia presentada en el I Seminario Internacional de Psicología en la Comunidad, 5-12 de setiembre, organizado por la Asociación de Psicólogos de Cuba, La Habana, Cuba.

Salvatierra, B. (1980b). Riesgos de la enseñanza del segundo idioma en la primera infancia. Ponencia presentada en el I Congreso Regional de Psicología, 30 de abril 5 de mayo, organizado por la Asociación de Psicólogos de Arequipa, Arequipa, Perú.

Salvatierra, B. (1981). Ludoterapia. Ponencia presentada en el I Seminario Regional de Psicología, 22 - 27 de junio, organizado por el Colegio de Psicólogos Consejo Directivo Regional III, Arequipa, Perú.

Salvatierra, B. (1983a). Influencia de la amígdala del lóbulo temporal sobre los fenómenos fásicos del sueño en el gato (félix domésticus). (Tesis de Maestría). Universidad Nacional Autónoma de México, México.

Salvatierra, B. (1983b). Neuropsicología. Arequipa: UNSA.

Salvatierra, B. (1984). La actividad póntico-genículo occipital (PGO) del sueño paradójico y su posible participación en la expresión e integración de las emociones. Ponencia presentada en el I Congreso Peruano de Psicología Clínica, 3 - 7 de diciembre, organizado por el Colegio de Psicólogos del Perú, Arequipa, Perú.

Salvatierra, B. (1986a). Neuropsicología. Arequipa: UNSA.

Salvatierra, B. (1986b). Consultando al psicólogo. Conciencia, 1(1), 4-11. 
Salvatierra, B. (1987). La enseñanza del segundo idioma en la primera infancia y sus repercusiones en la madurez para el aprendizaje escolar. Conciencia, 2(2), 11-22.

Salvatierra, B. (1989a). Neuropsicología. Arequipa: UNSA.

Salvatierra, B. (1989b). Neuropsicología. Manual de prácticas. Arequipa: UNSA.

Salvatierra, B. (1989c). Bases neuropsicológicas del aprendizaje. Segunda Especialidad en Psicología Educativa, Orientación y Consejería Escolar. Arequipa: Arequipa.

Salvatierra, B. (1991). Guía para la orientación vocacional y profesional. Arequipa: Publiunsa.

Salvatierra, B. (1992). ¿Qué hago? Arequipa: UNSA.

Salvatierra, B. (1993). El matrimonio. Arequipa: UNSA.

Salvatierra, B. (1995). Aportaciones el electroencefalograma al diagnóstico y tratamiento neuropsicológico de las dificultades del aprendizaje. (Tesis doctoral). Universidad Complutense de Madrid, España.

Salvatierra, B. (1997). Orientación psicológica para la familia. Arequipa: UNSA.

Salvatierra, B. (1998). Niños con dificultades de aprendizaje. Guía para padres, maestros y psicólogos. Arequipa: UNSA.

Salvatierra, B. (1999a). Asimetrías cerebrales y la emotividad y susceptibilidad del músico. Ponencia presentada en el IX Congreso Nacional de Psicología, 30 de setiembre - 2 de octubre, organizado por el Colegio de Psicólogos del Perú, Lima, Perú.

Salvatierra, B. (1999b). Asimetrías cerebrales y la emotividad y susceptibilidad del músico. Revista Peruana de Psicología, 4(7), 11-42.

Salvatierra, B. (2000). Orientación psicológica laboral. Arequipa: UNSA.

Salvatierra, B. (2001). Psicología del Minero. Arequipa: Formas Ediciones.

Salvatierra, B. (2003). Detección neuropsicológica de dificultades de aprendizaje en niños de edad escolar. Ponencia presentada en el I Congreso Nacional de Psicología Clínica - Educativa, 1 - 3 de agosto, organizado por el Colegio de Psicólogos Consejo Directivo Regional III, Arequipa, Perú.

Salvatierra, B. (2004). Cómo vivir la adolescencia sin padecerla. Guía para adolescentes, padres, maestros y psicólogos. Arequipa: Representaciones Gráficas S.C.R. Ltda.

Salvatierra, B. (2006). La dislexia a la luz de la neuropsicología evaluativa y la biocibernética. Ponencia presentada en el I Congreso Internacional de Psicología, 5 - 8 de julio, organizado por la Universidad Católica de Santa María, Arequipa, Perú.

Salvatierra, B. (2011a). Estudio del perfil psicológico de mineros peruanos entre 1998 y 2005. Revista de Psicología de Arequipa, 1(1), 82-100.

Salvatierra, B. (2011b). La orientación vocacional y profesional en el Perú: Repercusión ocupacional y productiva. Revista de Psicología de Arequipa, 1(2), 119-124.

Salvatierra, B. (2011c). Cultura de seguridad laboral para el minero. Investigaciones realizadas con mineros peruanos (1998-2008). Arequipa: Noceda. 
Salvatierra, B. (2013a). Influencia de la amígdala del lóbulo temporal sobre los fenómenos fásicos del sueño en el gato (feliz domesticus). Revista de Psicología de Arequipa, $3(2), 39-63$.

Salvatierra, B. (2013b). Detección neuropsicológica de dificultades del aprendizaje en niños de edad pre-escolar. Revista de Psicología de Arequipa, 3(2), 153-163.

Salvatierra, B. (2013c). El quehacer del psicólogo en la minería del siglo XXI. Ponencia presentada en el XVI Congreso Peruano de Psicología, 3 - 5 de octubre, organizado por el Colegio de Psicólogos del Perú, Arequipa, Perú.

Salvatierra B. (2014, 30 de junio). Comunicación personal. Arequipa, Perú.

Salvatierra, B. (2015). Hace treinta años se hizo realidad. Revista de Psicología de Arequipa, 5(1), 56-67.

Salvatierra, B., Quispe, D., \& Portugal, L. (1980). Un estudio con adolescentes usuarios ocasionales de cocaína. Ponencia presentada en el I Congreso Regional de Psicología, 30 de abril - 5 de mayo, organizado por la Asociación de Psicólogos de Arequipa, Arequipa, Perú.

Sos, R. y Esteban, C. (1998). Los comienzos de la psicología en Francia. En F. Tortosa Una historia de la psicología moderna (pp. 153-177). Madrid: McGraw-Hill.

Soto, M. y Arcos, M. E. (2010). Reserva cognitiva y rendimiento neuropsicológico en el adulto mayor arequipeño. Revista de Investigación, 1(1), 5-24.

Soto, M. y Belón, M. V. (2017). Indicadores de sensibilidad y especificidad para dos puntos de corte del Mini Mental State Examination: Estudio preliminar. Revista de Neuropsiquiatría, 80(2), 88-93.

Soto, M. y Cáceres, G. (2012). Funciones ejecutivas en adultos mayores alfabetizados y no alfabetizados. Revista Chilena de Neuropsicología, 7(3), 127-133.

Soto, M., Flores, G., \& Fernández, S. (2013). Nivel de lectura como medida de reserva cognitiva en adultos mayores. Revista de Neurología, 56, 79-85.

Ugarte y Chocano, E. (2014). Vida y arte en Belén Salvatierra y Alberto Vega. Arequipa: La Ciudad Producciones.

Valdivia, O. (1964). Historia de la psiquiatría peruana. Lima: s/e.

Valdivia, O. (1989). Panorama de la psiquiatría en el Perú. Lima: Universidad Nacional Mayor de San Marcos.

Vargas, F. (1966). El mundo sensorial del invidente. Revista de la Facultad de Letras, 3, 189-1999.

Vega, Z. (2011). Música en la catedral de Arequipa 1609-1881. Arequipa: Universidad Católica San Pablo. 